\title{
Combining sound and optic flow cues to reach a sound source despite lateral obstacles
}

\author{
F. Ruffier \\ Biorobotics \\ Institute of Movement Sciences \\ CNRS / Uni. of the Mediterranean \\ CP938, 163 ave. Luminy \\ 13288 Marseille France \\ franck.ruffier@univmed.fr
}

\author{
T. Mukai, H. Nakashima \\ Biologically Integrative Sensors \\ Bio-Mimetic Control, RIKEN \\ 2271-130, Anagahora \\ Nagoya, 463-0003 Japan \\ tosh@bmc.riken.jp \\ nakas@bmc.riken.jp
}

\author{
J. Serres and N. Franceschini \\ Biorobotics \\ Institute of Movement Sciences \\ CNRS / Uni. of the Mediterranean \\ CP938, 163 ave. Luminy \\ 13288 Marseille France \\ julien.serres@univmed.fr \\ nicolas.franceschini@univmed.fr
}

\begin{abstract}
In our project on the autonomous guidance of MicroAir Vehicles (MAVs) in confined indoor and outdoor environments, we have combined an insect vision-based autopilot with a directional sound sensor, with which a miniature hovercraft reaches a sound source along a corridor by automatically controlling its speed, its clearance from the walls, and its body yaw. A hovercraft is an air vehicle endowed with natural roll and pitch stabilization characteristics, in which planar flight control systems can be developed conveniently. Our hovercraft is fully actuated by two rear and two lateral thrusters. It travels at a constant altitude (about $2 \mathrm{~mm}$ ) and senses the obstacles by means of two lateral eyes that measure the right and left optic flows (OFs).

The visuo-motor control system, which has been previously called LORA III (Lateral Optic flow Regulation Autopilot, Mark III), is an insect-inspired dual OF regulator consisting of two interdependent feedback loops, each of which has its own OF set-point and controls its own translational degree of freedom (surge or sway).

The sound based control system servoes the robot course direction to the sound source direction estimated by an insect-inspired sound sensor.

Our computer-simulated experiments show that the hovercraft can navigate along a tapered corridor at a relatively high speed (up to $1.5 \mathrm{~m} / \mathrm{s}$ ). Both minimalistic visual and sound systems (comprised of only 4 pixels and two $0.1 \mathrm{~g}$ omni-directional microphones) suffices for the hovercraft to reach the target while controlling its clearance from the walls and its forward speed jointly, without any need for speed and range sensors.
\end{abstract}

Keywords: Biorobotics, sound, optic flow, navigation

\section{ACRONYMS}

\author{
OF Optic Flow \\ EMD Elementary Motion Detector \\ UAV Unmanned Air Vehicle \\ MAV Micro-Air Vehicle
}

\section{INTRODUCTION}

Insects are able to navigate swiftly in unfamiliar environments by extracting information from their own motion. The optic flow (OF) is the apparent motion of the image of contrasting features projected onto the insect's retina. Insects rely on OF to avoid collisions, to follow a corridor [1], [2], to control their flight speed [3], and to cruise and land [4], for example. Other insects such as male crickets rely on sound to perform phonotaxis by orienting their course to reach a mate [5].

Our latest autopilot, called LORA III, equips a hovercraft and consists of two interdependent $\mathrm{OF}$ regulators each of which has its own OF set-point [6]. The miniature hovercraft we are working on (Fig. 1), is an advantageous "MAV" in many ways. It makes no contact with the ground and "flies" on a plane at a constant height of about $2 \mathrm{~mm}$, which eliminates the need to implement an altitude control system on-board. A hovercraft is also endowed with inherent roll and pitch stabilization characteristics, which does away with the need to implement an attitude control system on-board. LORA III computer-simulated experiments showed excellent performances in controlling both forward speed and clearance from the walls in straight and tapered corridors [6]. In both types of corridor, the hovercraft managed to reach a safe forward speed and a safe clearance from the walls, which are commensurate with the lateral width of the corridor [6].

The sound sensors consist of two tiny $0.1 \mathrm{~g}$, omnidirectional microphones that we also used in our previous research on human-inspired sound source localization system [7]. Some robots have already been equipped with omnidirectional microphones [8]. Recently, Webb et al. showed how a simulated robot can control its course using both visual and sound cues [9].

The present account describes a simulated robot equipped with low cost vision sensors and low cost sound sensors, which avoids obstacles while reaching a sound source. The speed control and the wall avoidance systems have been previously designed and tested in simulation [6]. we add a novel feedback loop that makes use of a sound cue to make the robot reach a target.

In section 2, the simulated robot design is described including its sensors and actuators. Section 3 describes the model of the directional sound sensor used. In section 4 , the suggested autopilot combining sound and optic flow is described in detail. Section 5 deals with the computer-simulated experiments carried out on the automated hovercraft. The results show that the autopilot enables the robot to reach a sound source by wall-following or centring. It is concluded that the autopilot 
provides a simple, lightweight and low cost means of guiding a aerial vehicle.

\section{Design of the Simulated Robot}

\section{A. Simulation set-up}

All the present computer-simulated experiments were carried out on a standard PC equipped with the Matlab $^{T M} /$ Simulink software at a sampling frequency of $1 \mathrm{kHz}$ (for the vision based autopilot) and $100 \mathrm{kHz}$ (for the sound propagation and the sound directional sensor).

The visual contrasts are simulated using simulation tools previously developed in the lab [6], [10].

We have simulated the sound wave propagation to travel in the tapered corridors using the shortest path. The wave amplitude is assumed to decrease along the path as described by the following sound wave equation :

$$
S_{\text {Ear }}(t)=A \frac{1}{D_{\text {Ear } / \text { Source }}^{2}} e^{-j \omega_{0} \frac{D_{\text {Ear } / \text { Source }}}{V_{\text {SoundVelocityInAir }}}}
$$

with:

$\omega_{0}$ : the main frequency of the sound source,

$D_{\text {Ear/Source }}$ : the shortest distance between the robot's ear and the sound source,

$V_{\text {SoundVelocityInAir }}=340 \mathrm{~m} / \mathrm{sec}$ : the constant of the sound velocity in air.

In these first simulations, we neglect echos, sound wave reflections and wave guide effects.

\section{B. Actuators}

The robot is a miniature hovercraft that is rendered fully actuated in the plane by adding two lateral thrusters to the two rear thrusters (fig. 1). The robot can therefore move independently in any direction in the plane along its 3 degrees of freedom :

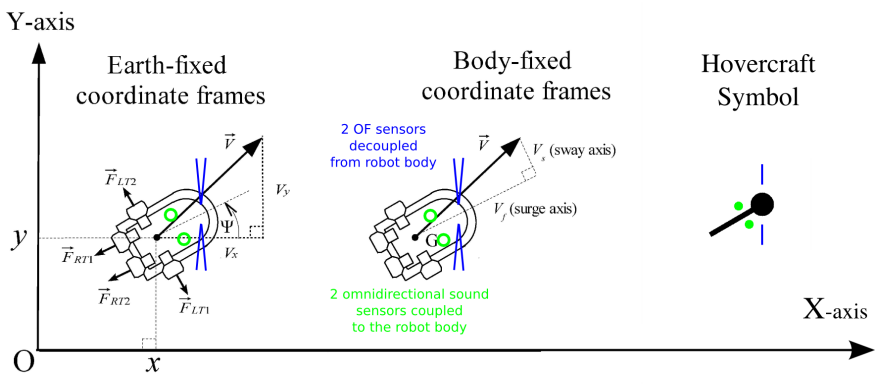

Fig. 1. Description of the simulated robot . The hovercraft is equipped with two side and two rear thrusters that acts upon the forward thrust via the sum of the rear thrusts $F_{R T 1}+F_{R T 2}$, upon the yaw torque via the difference between the left and right rear thrusts $F_{R T 1}-F_{R T 2}$ and upon the side thrust via the difference between the left and right thrusts $F_{L T 1}-F_{L T 2}$. The sound sensors are mounted on the robot body (Green circles) while the right and left gazes of optic flow sensor are uncoupled from the robot's body (Blue lines). In the plots of the simulated trajectories, the robot is represented by the symbol shown to the right.
- The two rear thrusters generate forward motion when driven in concert, and yaw motion when driven differentially,

- The two lateral thrusters generate lateral thrust leading to side-slip motion in the robot local coordinates,

- A micro servomotor allows the visual sensor platform to be uncoupled from the robot's body.

\section{Sensors}

Figure 1 schematics show that the simulated robot is equipped with:

- Two omni-directional microphones attached to the robot body that feed a directionally sensitive cricket-inspired model,

- Two optic flow sensors looking to the right and to the left respectively, whose model has been inspired from those of flying insects.

Each OF sensor consists of two photoreceptors (two pixels) driving an Elementary Motion Detector (EMD). The visual axes of the two photoreceptors are separated by an interreceptor angle: $\Delta \phi=4^{\circ}$. Each photoreceptor angular sensitivity is a bell-shaped function with an acceptance angle (angular width at half height) of similar value: $\Delta \rho=4^{\circ}$. The principle of the EMD circuit used here was derived from electrophysiological experiments performed in houseflies [11], [12]. It does not belong to the "Reichardt correlator" scheme [13] but belongs rather to the "token-matching schemes". The nonlinear circuit is driven by two neighbouring channels, each of which involves several processing stages, some of which are realized in a microcontroller [14]. The EMD output is a monotonic function of the $\mathrm{OF}$ within a 10-fold range (from $40^{\circ} / \mathrm{s}$ to $400^{\circ} / s$ ) [15]. Whenever the EMD circuit does not detect any new contrasting features, it holds the last measured value for a period of $0.5 \mathrm{~s}$.

The sound sensors are fixed to the robot body while the optic flow sensors are uncoupled (in yaw) from the robot's body. The gazes of the optic flow sensors are maintained fixed with respect to the ground-based coordinates, by means of a gyrobased heading-look system.

\section{BIO-INSPIRED DIRECTIONAL SOUND SENSOR}

Figure 2 shows the whole model of directionally sensitive sound sensor. This model is inspired by the cricket ear and based on Webb et al' previous studies in 2000 [16], [9]. We

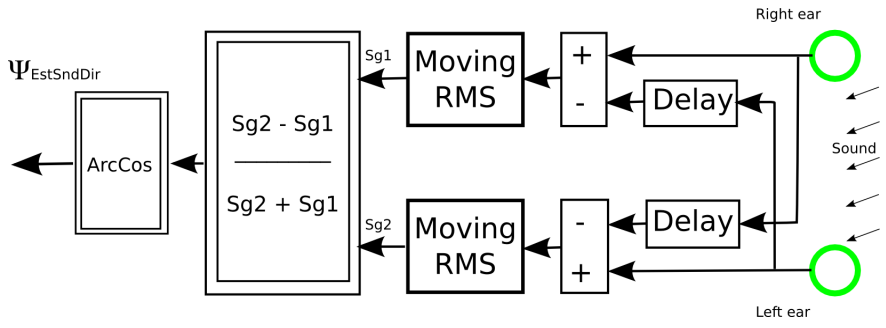

Fig. 2. Bio-inspired directional sound sensor model. The sound sensor model is derived from cricket ear, studied by Webb et al in 2000 [16], [9]. 


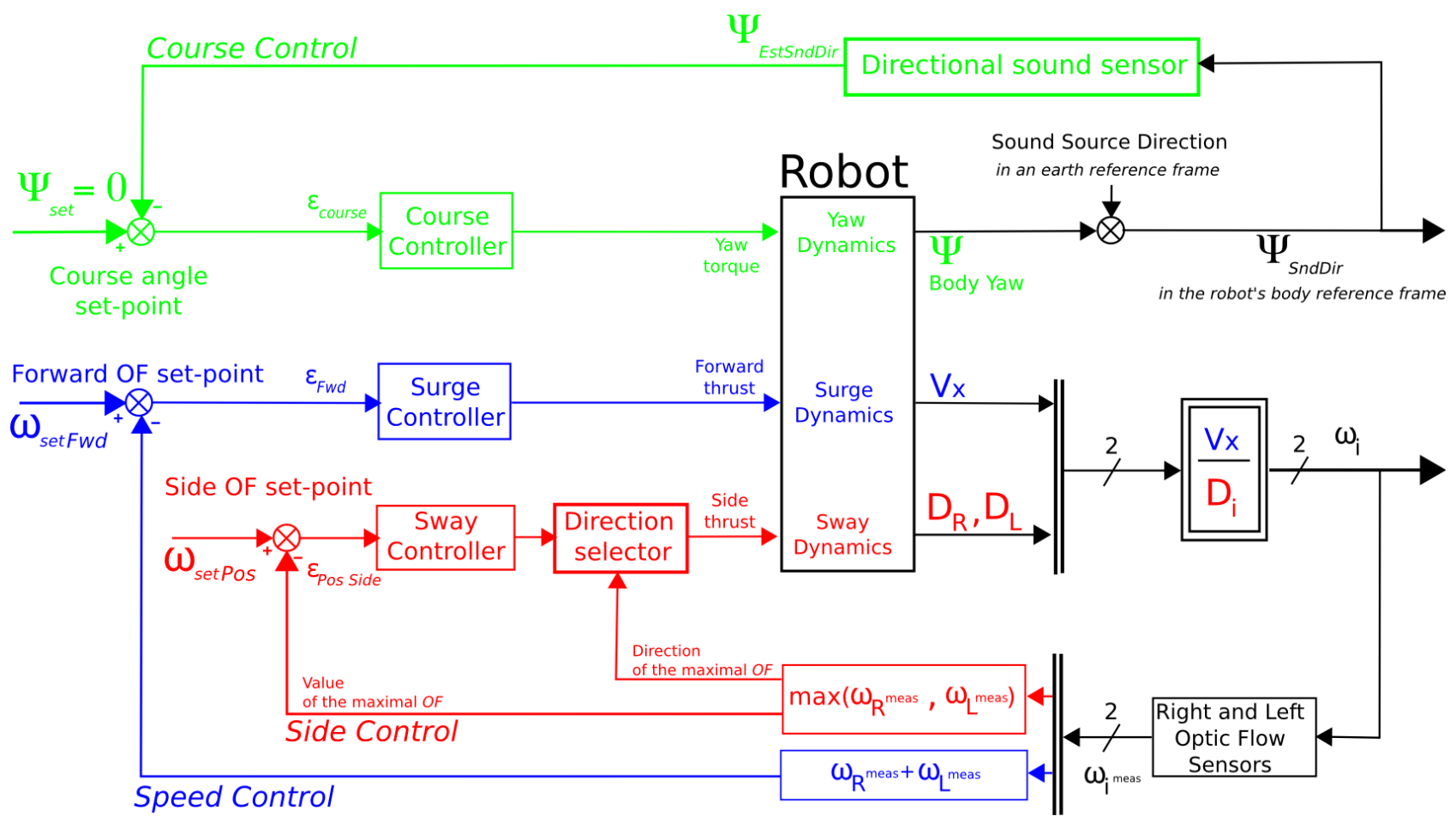

Fig. 3. Simulated autopilot. This control scheme describes how the simulated robot (i) can reach a sound source by acting upon its course, (ii) can control its speed by acting upon the forward thrust, and (iii) can control its side positioning by acting upon the side thrust.

assume that the robot's ear system is tuned to carrier frequency of the sound source, which is $4.7 \mathrm{kHz}$ (the same frequency as the cricket song). The two first steps of the directional sound sensor model use data related to the carrier frequency value. The simulated sound directional sensor is based on two very light omni-directional microphones (weight $0.1 \mathrm{~g}$ ).

This model involves six steps:

1) Spatial separation between right and left ears : the distance corresponds to one quarter of the sound source wavelength. In case the sound source is placed on the side of the robot, this step makes the phase between the two ear signals delayed by $\pi / 2$.

2) Temporal delay of one quarter of the sound source wave period on each ear signal. In case the sound source is placed on the side of the robot, this step produces $\pi$ phase difference between ear signal and delayed signal.

3) Subtraction between ear signal and delayed signal.

4) Moving average using the Root Mean Square (RMS) method (the number of samples that are averaged correspond to several sound source wavelengths): the output signal gives the mean amplitude of the subtracted signals,

5) Ratio between difference and sum : this gives the direction of the sound source,

6) Nonlinear function ArcCos : it gives the angular direction of the sound source with respect to the robot body.

To challenge the sound sensor's model, we simulated the noisy sound originating in the motor/duct fan assembly that produces the hovercraft lift. We added both a white noise and a sinusoidal signal $(1 \mathrm{kHz})$ of constant amplitude to the left and right ear signals.

The adverse effect of the noisy sound produced by the robot main motor, was dramatically reduced by adding a bandselective Butterworth filter between steps 1 and 2 (6th-order filter between $4 \mathrm{kHz}$ and $5.4 \mathrm{kHz}$ ).

In addition, we introduced a low-pass filtering stage on each sound sensor's output to reduce the high frequency fluctuation caused by sampling and noise (first order filter at the cutting frequency of $16 \mathrm{~Hz}$ ).

\section{Autopilot MAKing THE ROBOt REACH A SOUND SOURCE DESPITE LATERAL OBSTACLES}

Figure 3 shows that the autopilot equipping the simulated robot is composed of 3 feedback loops:

1) Speed control: the sum of the right and left Optic Flow is maintained constant by means of an optic flow regulator that controls the robot's speed [6]. This feedback loop makes the robot automatically travel at a ground speed $v_{x}$ proportional to the corridor width without measuring (or estimating) the local corridor width and the speed.

2) Side positioning control: the maximal Optic Flow (right or left) is maintained constant by means of an optic flow regulator that controls the robot's side thrust. This feedback loop makes the robot automatically travel at a distance from the nearest wall, $D_{L}$ or $D_{R}$, that is proportional to the speed without measuring (or estimating) the corridor width and the speed [6]. A control 
direction selector automatically selects the wall that will be followed.

3) Course control (or Body orientation control): the robot's body automatically orients in the direction of the sound source by means a feedback loop that controls the robot's yaw. This feedback loop will make the robot reach the sound source automatically.

The tuning procedures for both speed and side controllers is described in detail in [6].

\section{Simulation Results}

To test the performance of our robot equipped with such autopilot, we simulated its travel along a tapered corridor for different initial positions and different sound source positions. The visual environment simulated here is a 12-meter long tapered corridor with a 2-meter wide entrance and a 0.5 -meter wide constriction located midway. Its right and left walls are lined with the same random pattern of grey vertical stripes as that used previously [6] (covering a 1-decade contrast range from $4 \%$ to $38 \%$, and a 1.5 -decade angular frequency range from $0.034 \mathrm{c} /{ }^{\circ}$ to $1.08 \mathrm{c} /{ }^{\circ}$ reading from the longitudinal axis of the corridor).

Irrespective of its initial position, the hovercraft can be seen to automatically slow down as it approaches the narrowest section and to accelerate again beyond the constriction (Figure $4 b)$. The hovercraft therefore negotiates a narrowing passage by automatically decelerating.

The present robot trajectories have been recorded using
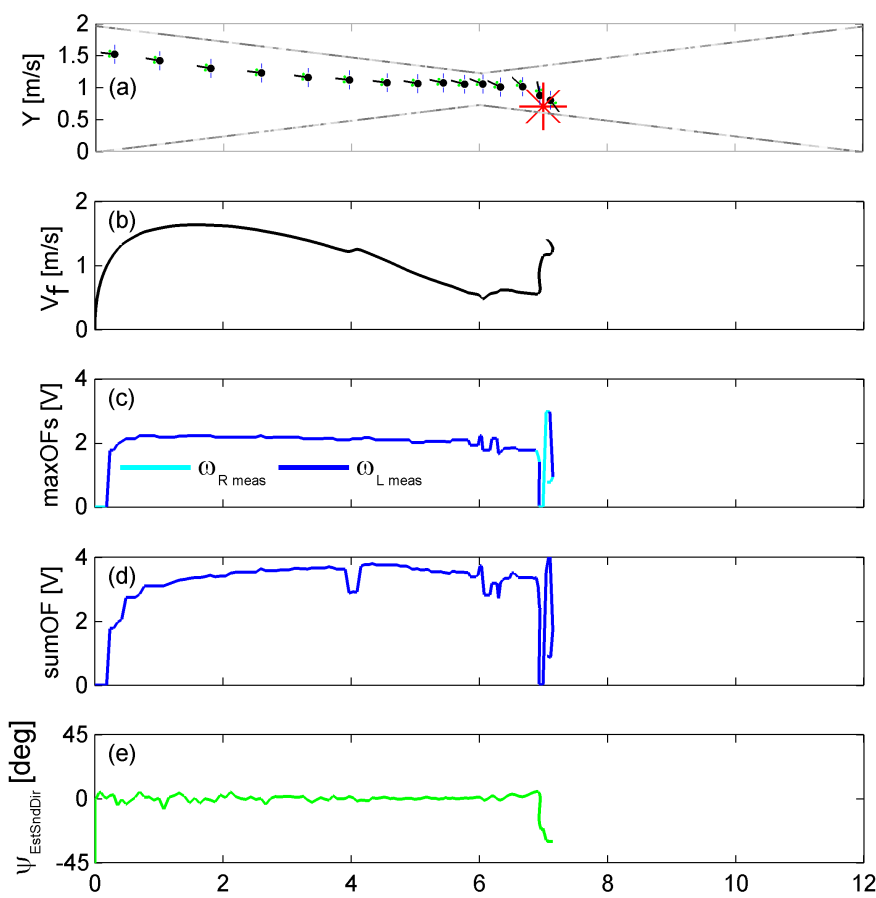

Fig. 4. Robot's flight path in a tapered corridor. The robot starts near the left wall and reaches the sound source placed near the right wall just after the constriction. The robot can be seen to follow the left wall, and decelerate. The robot starts turning toward the sound source at about the narrowest section. exactly the same autopilot parameters. The side OF set-point is set to $\omega_{\text {setSide }}=2.21 \mathrm{~V}$ corresponding to $230^{\circ} / \mathrm{sec}$ and the forward OF set-point is set to $\omega_{\text {setF } w d}=3.28 \mathrm{~V}$ corresponding to $300 \%$ sec.

In every trajectories (Fig. 4 to 8 ), the speed control and side control loops, respectively, strive to maintain the sum and the larger value between right and left optic flows constant all the way to the sound source (e.g. Fig. 4 d,c). In addition, the third (sound based) control loop also strives to maintain the robot's heading oriented towards the sound source during the final approach. As the robot gets closer to the target, the sound sensor output signal becomes more precise despite the noise produced by the main motor.

\section{CONCLUSION}

We have presented here five computer-simulated trajectories of the robot performing speed control, side control and course control to reach a sound source despite lateral obstacles.

Our simulation shows that a robot can reach a sound source using solely insect-inspired sound and optic flow sensing. Our autopilot is rather simple in terms of processing and rises exclusively passive (non-emissive) sensors that are low cost, light weight and power-lean.

Future investigation should try to combine vertical and horizontal sound-based control system to make a robot reach a target.

Our autopilot could be nevertheless applied to vehicles in
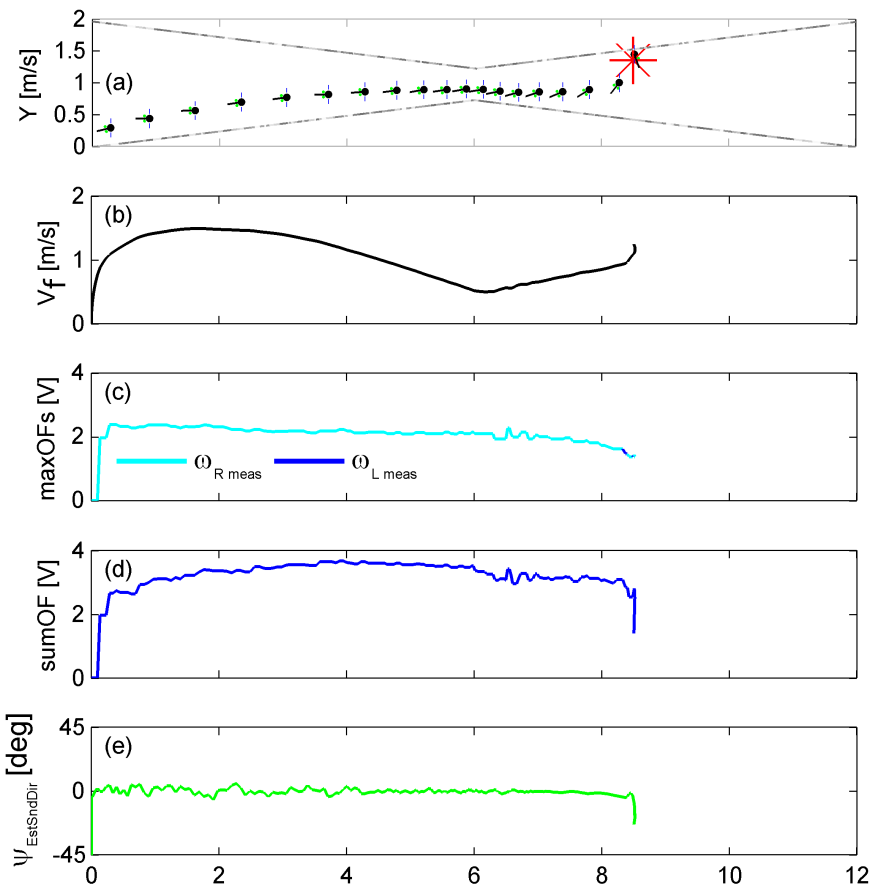

Fig. 5. Second simulated robot trajectory. The robot starts near the right wall and reaches the sound source located on the opposite wall in the middle of the widening part of the corridor. The robot can be seen to follow the right wall and decelerate when the corridor width decreases. The robot starts turning toward the sound source at about the narrowest section. 

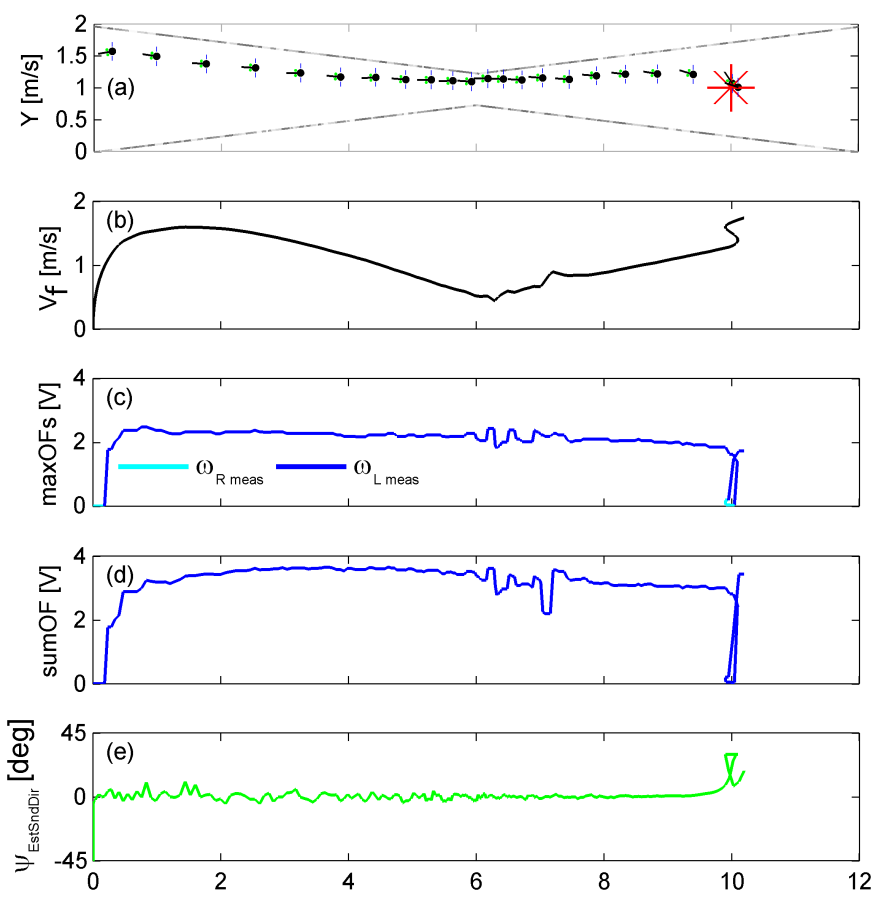

Fig. 6. Third simulated robot trajectory. The robot starts near the left wall and reaches the sound source inside the second part of the corridor.
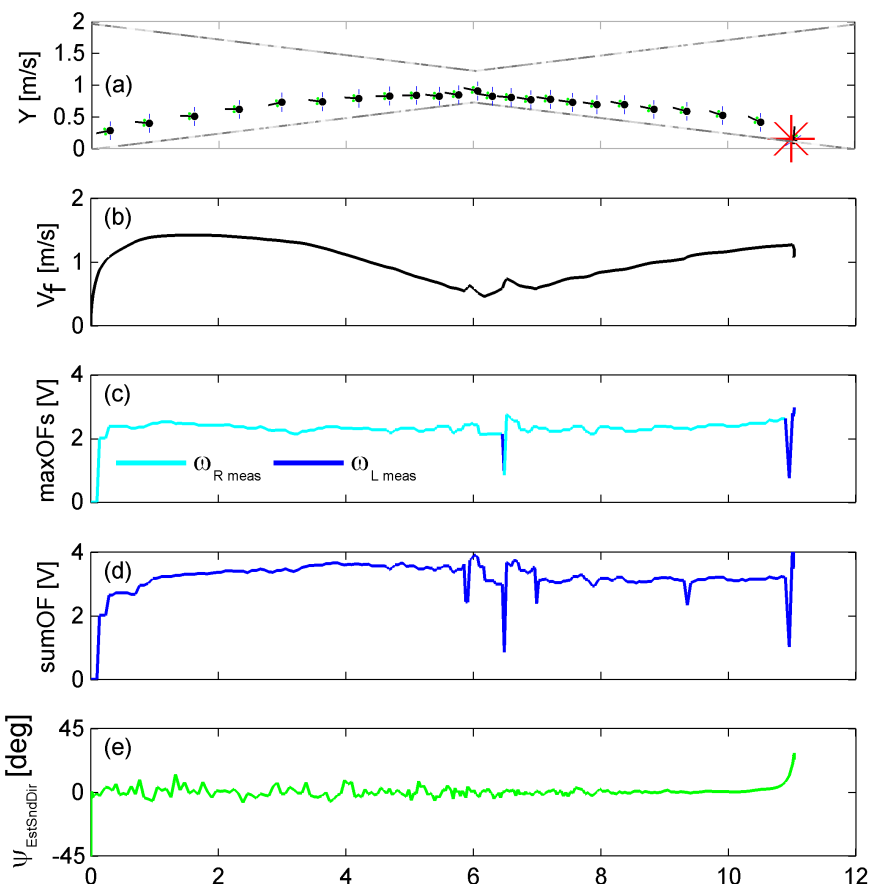

Fig. 7. Fourth simulated robot trajectory. The robot starts near the right wall and reaches the sound source closed to the right wall, near the extremity of the corridor.

which the yaw, surge and sway dynamics are uncoupled such as MAVs (e.g.: conventional, coaxial, or quadrotor minihelicopters).

Insect-based sensory-motor control systems can yield solutions
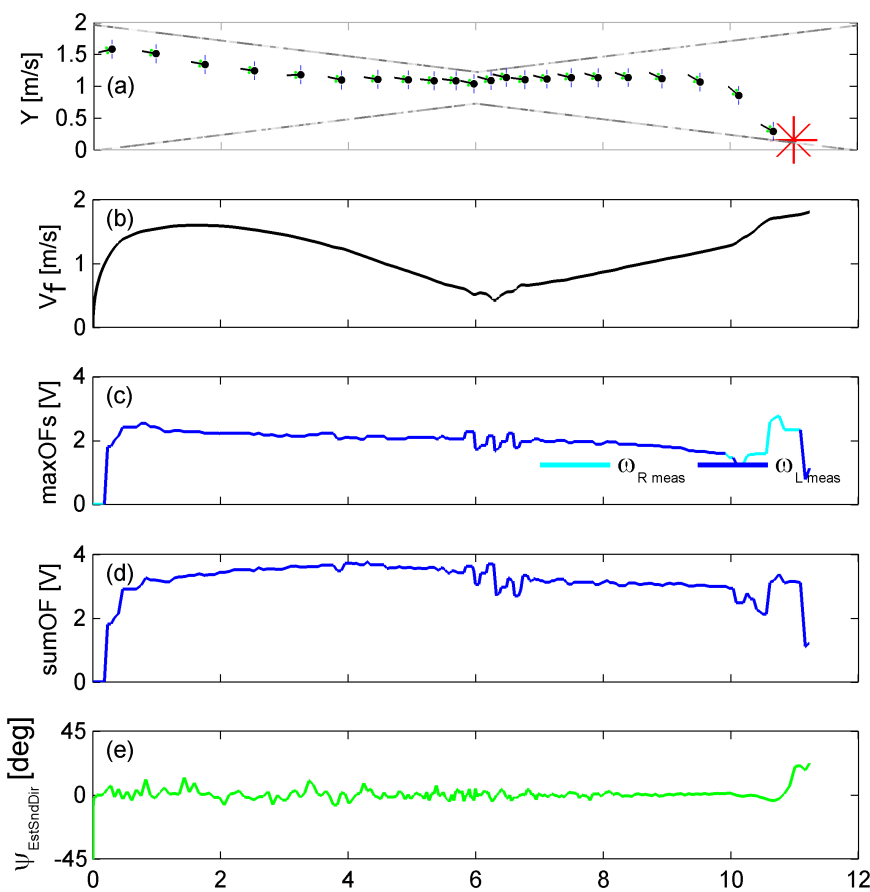

Fig. 8. Fifth simulated robot trajectory. The robot starts near the left wall and reaches the sound source closed to the right wall, near the extremity of the corridor.

requiring a much smaller number of sensors (here only 4 pixels and 2 tiny microphones) than those currently harnessed to mobile robots. The autopilot presented here may open the way to lightweight and low-cost visual guidance systems for autonomous vehicle navigation in unfamiliar indoor environments, as well as in urban canyons where GPS signals may be considerably attenuated by the presence of buildings. The non-emissive OF sensors and the simple processing system described are particularly suitable for use on MAVs, whose small size imposes draconian constraints on avionic payload and onboard energy resources.

\section{ACKNOWLEDGMENT}

The authors thank S. Viollet and E. Ogam for their fruitful comments and suggestions during this work. This work was supported by CNRS, by the Univ. of the Mediterranean, by RIKEN (Bio-Mimetic Research Center, Nagoya) and by JSPS via the Invitation Fellowship Programs for Research in Japan.

\section{REFERENCES}

[1] W.H. Kirchner and M.V. Srinivasan, "Freely flying honeybees use image motion to estimate object distance," Naturwissenschaften, vol. 76, pp 281-282, 1989.

[2] J. Serres, G.P. Masson, F. Ruffier, and N. Franceschini, "A bee in the corridor: centering and wall-following," Naturwissenschaften, 2008 (in rev.).

[3] M.V. Srinivasan, S.W. Zhang, M. Lehrer, and T.S Collett, "Honeybee navigation. en route to the goal: visual flight control and odometry," J. of Experimental Biology, vol. 199, pp. 237-244, 1996.

[4] N. Franceschini, F. Ruffier, and J. Serres, A bio-inspired flying robot sheds light on insect piloting abilities, Current Biology, vol. 17, pp. 329-335, 2007 
[5] K. Schildberger, "Behavioural and neuronal methods of cricket phonotaxis," Experentia (Basel) 44:408-415, 1988.

[6] J. Serres, D. Dray, F. Ruffier, and N. Franceschini, ”A vision-based autopilot for a miniature air vehicle: joint speed control and lateral obstacle avoidance," Autonomous Robots 25(1-2), 103-122, 2008

[7] H. Nakashima, and T. Mukai, "3D Sound Source Localization System Based on Learning of Binaural Hearing," Proceedings of 2005 IEEE International Conference on Systems, Man and Cybernetics (IEEE SMC 2005), 3534-3539, 2005.

[8] J. Huang , N. Ohnishi and N. Sugie, "Building ears for robots: Sound localization and separation," Artificial Life and Robotics, 1(4):157-163, 1997.

[9] B. Webb, and R. Harrison, "Integrating sensorimotor systems in a robot model of cricket behaviour," Sensor Fusion and Decentralized Control in Robotic Systems III, Editors: McKEE GT, Schenker PS, Proceedings of the Society of Photo-Optical Instrumentation Engineers (SPIE), vol. 4196, pp: 113-124, 2000.

[10] F. Ruffier, "Pilote automatique Biomimetique," PhD Thesis, Institut National Polytechnique (INP), Grenoble, 2004.

[11] N. Franceschini, A. Riehle, and A. LeNestour, "Directionally selective motion detection by insect neurons," In: Stavenga DG, Hardie RC (eds) Facets of vision. Springer, Berlin Heidelberg New York, pp 360-390, 1989.

[12] C. Blanes, "Appareil visuel élémentaire pour la Navigation à vue d'un robot mobile autonome," Master Thesis (DEA in French), Neurosciences, Univ. Aix-Marseille II., 1986.

[13] W. Reichardt, "Movement perception in insects," In: Processing of Optical Data by Organisms and by Machines (ed. W. Reichardt), pp. 465-493. New York: Academic Press, 1969.

[14] F. Ruffier, S. Viollet, S. Amic, and N. Franceschini, "Bio-inspired optical flow circuits for the visual guidance of micro air vehicles," Proc. of Int Conf IEEE ISCAS, Bangkok (3):846-849, 2003.

[15] F. Ruffier and N. Franceschini, "Optic flow regulation: the key to aircraft automatic guidance," Robotics and Autonomous Systems, vol. 50(4), pp. 177-194, 2005.

[16] B. Webb and T. Scutt, "A simple latency-dependent spiking-neuron model of cricket phonotaxis," Biological Cybernetics, vol. 82, pp: 247$269,2000$. 